\title{
As práticas dos centros comunitários para idosos e a promoção do envelhecimento ativo: uma revisão sistemática
}

\author{
The senior centers practices and the promotion of active ageing: a systemic review
}

\author{
Ivani Soleira Gomes* \\ Simone Caldas Tavares Mafra**
}

\section{Resumo}

O presente estudo objetivou analisar as contribuições das ações desenvolvidas nos centros comunitários para idosos na perspectiva do envelhecimento ativo. Como método buscou-se estudos nas bases de dados LILACS (Literatura Latino-Americana e do Caribe), Medline (Medical Literature Analysis and Retrieval System Online), SciELO (Scientific Eletronic Library Online) e Web of Science, os quais envolvessem a população dos centros comunitários de idosos e fossem voltados para qualidade de vida e promoção da saúde, aspectos constituidores do envelhecimento ativo. Essa pesquisa resultou em 17 trabalhos encontrados que acionaram conceitos afetos aos pilares do envelhecimento ativo, que, por sua vez, estão imbricados com a qualidade de vida e promoção da saúde. Os resultados dos estudos que envolveram idosos integrantes de centros comunitários para idosos evidenciaram que o referido equipamento tem contribuído para a efetivação dos princípios do envelhecimento ativo. Contudo, ainda faltam análises que demonstrem a sua efetividade e, quando necessário, indiquem pontos a serem corrigidos a partir da retroalimentação dos dados gerados pelas pesquisas, fornecendo fundamentos para elaboração de uma espécie de diretriz e correção de ações para esses espaços.

Palavras-chave: Envelhecimento. Envelhecimento ativo. Centro comunitário para idosos. Saúde. Qualidade de vida.

\begin{abstract}
This study aims to analyze the contribution of the actions developed in the senior centers in the view of active ageing. The method used was to search for studies on the LILACS, Medline, SciELO and Web of Science databases, involving the population of community centers, focused on aspects of quality of life and health promotion and active ageing constitutors. This research resulted in 17 articles that triggered the affection concepts to the pillars of active ageing which are interwoven with the quality of life and promoting health. It was concluded that the studies involving elderly members of senior centers showed that such equipment has contributed to the implementation of the principles of active ageing. However, there is a lack of analyses that demonstrate its effectiveness and, when necessary, indicate issues which need to be corrected from the feedback of the data generated by surveys, providing grounds for drawing up some kind of guideline and correction of actions for these spaces.
\end{abstract}

Keywords: Ageing. Active ageing. Senior center. Health. Quality of life.

\footnotetext{
* Tecnóloga em Cooperativismo da Universidade Federal de Viçosa - UFV. Mestre em Economia Doméstica. Doutoranda em Economia Doméstica pela UFV.

** Professora Titular da Universidade Federal de Viçosa (UFV). Professora no Programa de Pós-graduação em Economia Doméstica da UFV. Doutora em Engenharia de Produção pela Universidade Federal de Santa Catarina. Pós doutorado em Minority Aging pela University of Texas Medical Branch, Texas, EUA.
} 


\section{Introdução}

O envelhecimento populacional faz parte da realidade de muitas nações no mundo, sendo que, cada país, ao seu modo, vivencia o envelhecimento populacional de acordo com os seus contextos culturais, sociais, políticos e econômicas. Kalache, Veras e Ramos (1987) mencionam a universalidade do fenômeno como resultado de uma transição demográfica mundial iniciada no século XX.

Nos países desenvolvidos, o fenômeno tem se dado mais lentamente. Na França, por exemplo, o quantitativo de idosos aumentou de 10\% para 20\% em 150 anos; já em países como Brasil, China e Índia, a mudança poderá ser verificada em 20 anos (REIS; BARBOSA; PIMENTEL, 2016). O ritmo mais ou menos acelerado do processo de envelhecimento impõe realidades distintas às nações, dado que quanto mais demoradamente se der o processo, maior será o tempo para adequação e organização das instituições diante da nova dinâmica demográfica. Nesse sentido, os países em vias de desenvolvimento encontram-se menos preparados nesse cenário de população envelhecida (BARBOSA et al., 2017).

No Brasil, o incremento da população de idosos destaca-se como uma das principais mudanças nos últimos 100 anos. Nesse período, a expectativa de vida saltou de 33,5 anos em 1910, para 73 anos, em 2009. Em paralelo, é observado o decréscimo nas taxas de fecundidade, resultando, assim, em um aumento proporcional na população de idosos em comparação ao número de crianças (MINAYO, 2012).

Esse cenário tem modificado a estrutura etária brasileira, de modo que, além da diminuição na base da pirâmide etária, haverá o alargamento na extremidade superior, evidenciando o envelhecimento da população idosa, ou seja, o quantitativo de pessoas com 80 anos ou mais aumentou e aumentará em comparação ao de jovens e crianças (MINAYO, 2012). O quantitativo de idosos permanecerá crescendo, de sorte que para cada grupo de 100 crianças ( 0 a 14 anos) haverá 47,7 indivíduos com 65 anos, e mais em 2020, chegando a 113,2 e 160,90 nos anos de 2040 e 2050, respectivamente (REIS; NORONHA; WAJNMAN, 2016).

Paralelamente às mudanças na estrutura demográfica, o Brasil também experimenta o processo de transição epidemiológica, a qual marca a alteração no perfil de índices elevados de mortalidade, em decorrência de doenças infectocontagiosas para um padrão de mortes resultantes de patologias crônico-degenerativas (REIS; NORONHA; WAJNMAN, 2016). Nesse caso, o aumento da população idosa vem acompanhado pelas mudanças no perfil epidemiológico e nutricional, registrando-se um predomínio das doenças crônicas não 
transmissíveis.

Uma vez instalado o fenômeno do envelhecimento populacional, medidas devem ser tomadas na perspectiva de garantir que esse processo aconteça com boa qualidade de vida (VERAS, 2012). Contudo, há um consenso que o conceito de qualidade de vida é amplo e de difícil operacionalização. Sua construção envolve saúde, visão de mundo, aspectos psicológicos, os modos de se relacionar em sociedade, entre outros (MARQUES, 2017). A Organização Mundial de Saúde (OMS) define qualidade de vida como:

[...] grau de satisfação das necessidades da vida humana - como alimentação, acesso à água potável, habitação, trabalho, educação, saúde, lazer e elementos materiais que tem como referência noções subjetivas de conforto, bem-estar e realização individual e coletiva (BRASIL, 2012, p. 29).

O entendimento de que a saúde figura como protagonista na qualidade de vida e do reconhecimento da importância da manutenção da participação dos idosos nas atividades como forma de mantê-los inseridos socialmente mobilizou organismos como a Organização Mundial de Saúde (OMS) e a Organização Pan-Americana (OPAS), entre outros. A OMS organiza-se para compreender as dinâmicas impostas por uma população envelhecida e, assim, construir um quadro propositivo para as questões decorrentes da nova realidade demográfica, tendo, a partir dos anos 1990, reorientado suas políticas, sugerindo que as ações no campo da saúde deveriam ser direcionadas para o chamado envelhecimento ativo (YAMAMOTO, 2010).

Nesse movimento, a OMS produz o documento “Envelhecimento Ativo: Uma Política de Saúde", no qual define o envelhecimento ativo como um "processo de otimização das oportunidades de saúde, participação e segurança, com o objetivo de melhorar a qualidade de vida à medida que as pessoas ficam mais velhas (OMS, 2005, p. 13). O documento da OMS defende que a inserção social do idoso deve extrapolar as atividades de caráter econômico. Diz respeito às várias possibilidades de interações interpessoais do idoso na comunidade, na família e na economia (PEDRO, 2013).

Com isso, a promoção do envelhecimento ativo redimensiona o olhar para a velhice, colocando em cena novos papéis aos indivíduos, comunidades, sociedade e governos. Nesse sentido, a abordagem do envelhecimento sinaliza para a importância do envolvimento dos indivíduos em atividades promotoras de bem-estar físico, mental e social em todo ciclo vital e sugere responsabilidades no âmbito individual, social e governamental (AZEVEDO, 2015). A 
política inicialmente foi desenvolvida a partir dos pilares participação, saúde e segurança e, mais recentemente, foi incluída a dimensão aprendizagem ao longo da vida (CILB, 2015).

O envelhecimento ativo, preconizado pela OMS, tem orientado e subsidiado as nações na elaboração de políticas focadas para as diferenças do envelhecimento populacional (VELOSO, 2015). E, assim, inúmeras iniciativas de organismos públicos e da sociedade civil vêm efetivando suas ações para criação de espaços que promovam a convivência e participação social, a aprendizagem e a saúde, de modo que favoreçam a qualidade de vida das pessoas idosas. Dentre essas iniciativas destacam-se os espaços como os centros de convivência, centros-dia, centros comunitários para idosos, universidades abertas da terceira idade e grupos de idosos vinculados ao Programa Estratégia Saúde da Família, entre outros.

Os centros de convivência para idosos vem se ocupando de ações voltadas para a população de pessoas idosas em países como França, Inglaterra, China, Irlanda, Espanha, Canadá, Estados Unidos. No Brasil, os trabalhos educativos para idosos iniciaram-se com o Serviço Social do Comércio (Sesc), com ações voltadas ao lazer, seguidas pelas universidades, a partir da década de 1980, considerando as intervenções na área do ensino, saúde e lazer (MOURA; VERAS, 2017). Também compõe esse cenário o serviço de convivência e fortalecimento de vínculos, previsto pelas portarias nํ 46/SMADS/2010 e nำ09/SMADS/2012, do então Ministério do Desenvolvimento Social - MDS (FRANCISCO; PINHEIRO, 2018).

O centro-dia para idosos foi viabilizado pelo Sistema Único de Assistência Social (SUAS) com a função de acolher pessoas idosas e seus familiares em situação de dependência. Esses espaços, sob diversas nomeações - tais como, centro de convivência, centro comunitário, grupo de convivência, casa-dia, universidade aberta à terceira idade constituem-se como locais qualificados de atuação no processo de ressignificação e qualificação da experiência de envelhecer, por meio de ações promotoras da saúde, educação e socializadoras, criando um espaço favorável ao desenvolvimento da autonomia e da participação, com perspectiva de repercutir na qualidade de vida dos idosos e de seus familiares (YAMAGUCHI et al., 2018).

Nesse sentido, esses centros são estratégias locais que podem produzir efeitos positivos na região, estado, país e, ou, internacionalmente no que tange ao compromisso da sociedade, organizações e governos com a qualidade de vida desse estrato populacional. A importância desse equipamento também é reconhecida em países como os Estados Unidos, dado que, há mais de 70 anos, é referência na oferta de serviços de alimentação, saúde, 
socialização, lazer, cidadania e educação (GIUNTA et al., 2012). De um modo geral, esses grupos promovem atividades físicas, educativas e promotoras de saúde que visam o desenvolvimento de habilidades que favorecem a autonomia e o envelhecimento com qualidade (MARTINS; CASETTO; GUERRA, 2019).

Diante da importância do papel desses espaços de convivência na construção de uma velhice saudável, torna-se relevante conhecer a dinâmica desses espaços, bem como as estratégias adotadas para o alcance da qualidade de vida, promoção da saúde e interação social. Nesse sentido, este estudo objetivou analisar as práticas dos centros convivência para idosos na perspectiva da promoção do envelhecimento ativo.

\section{Métodos}

Trata-se de um estudo de revisão sistemática, conduzido a partir da metodologia descrita no Preferred Reporting Items for Systematic Reviews and Meta-Analyses - PRISMA (MOHER et al., 2009), tendo como foco a análise da produção científica acerca das práticas dos centros comunitários para idosos na promoção do envelhecimento ativo. O estudo foi orientado para responder como o envelhecimento ativo tem fundamentado as práticas nos centros comunitários para idosos, tendo como objetivo analisar as contribuições das ações desenvolvidas nos programas destinadas à pessoa idosa para o envelhecimento ativo. A formulação da pergunta considerou o acrônimo PICOS (população, intervenção, controle, resultados/outcomes e tipo de estudo/contexto), conforme quadro 1.

Quadro 1 - Descrição da estratégia PICOS

\begin{tabular}{|c|c|}
\hline Acrônimo & Descrição \\
\hline P & Centros comunitários para idosos \\
\hline I & Práticas dos centros comunitários para idosos \\
\hline C & Política do envelhecimento ativo \\
\hline O & Promoção do envelhecimento ativo \\
\hline S & Observacional \\
\hline
\end{tabular}

Ainda que a sistematização do conceito do envelhecimento ativo tenha ocorrido nos anos 2000, não houve restrição quanto às datas das publicações, uma vez que se considerou a possibilidade da identificação de ações fundamentadas na saúde, educação, segurança e 
participação. A pesquisa foi realizada no dia 25 de maio de 2019 utilizando os descritores base de dados dos Descritores em Ciências da Saúde (DeCS): "quality of life" and "aging" and "health policy" and "healthy aging" or "senior centers".

A pesquisa foi realizada nos bancos de dados LILACS (Literatura Latino-Americana e do Caribe), Medline (Medical Literature Analysis and Retrieval System Online) e SciELO (Scientific Eletronic Library Online) e Web of Science. Na base Web of Science, a procura restringiu-se à coleção principal como estratégia de busca. Os dados foram recolhidos de forma independente por dois pesquisadores, e as discordâncias entre estes foram resolvidas por consenso pelos revisores.

A busca por um envelhecimento, tal como preconizado pela OMS, perpassa ações voltadas para a manutenção e, ou, melhoria da qualidade de vida, que, por sua vez, está associada à saúde. Nesse sentido, os esforços devem ser direcionados para implementação de ações que visem manter e, ou, minimizar perdas de capacidade funcional, do mesmo modo medidas curativas, preventivas e promotoras de saúde (VERAS, 2012a). Assim, a inclusão dos artigos obedeceu aos seguintes critérios: (1) "Trabalhos desenvolvidos com idosos de centros comunitários para idosos" e "Trabalhos resultantes de ações para manutenção, prevenção e promoção da saúde", ou (2) "Trabalhos desenvolvidos com idosos de centros comunitários para idosos" e "Trabalhos que discutem qualidade de vida dos idosos".

Foram excluídos os trabalhos que envolveram pessoas com idade inferior a 60 anos de idade; avaliação física, nutricional, psicológica e cognitiva; avaliação e, ou, implementação de programa (análise ergonômica e, ou, de equipamentos); desenvolvimento, avaliação e, ou, validação de instrumento de avaliação; foco em doença - estudos de prevalência/predição/correlação, identificação e análise de sintomas de doenças, utilização de medicamento/suplemento; formação profissional. Também foram excluídos as teses, dissertações e trabalhos realizados a partir de fontes secundárias (relatório, reflexão, revisão de bibliografia). A figura 1, a seguir, apresenta a síntese do processo de seleção dos trabalhos.

Figura 1 - Fluxograma de identificação e seleção dos artigos para revisão sistemática sobre o envelhecimento ativos e os centros comunitários para idosos. Viçosa-MG, 2019. 


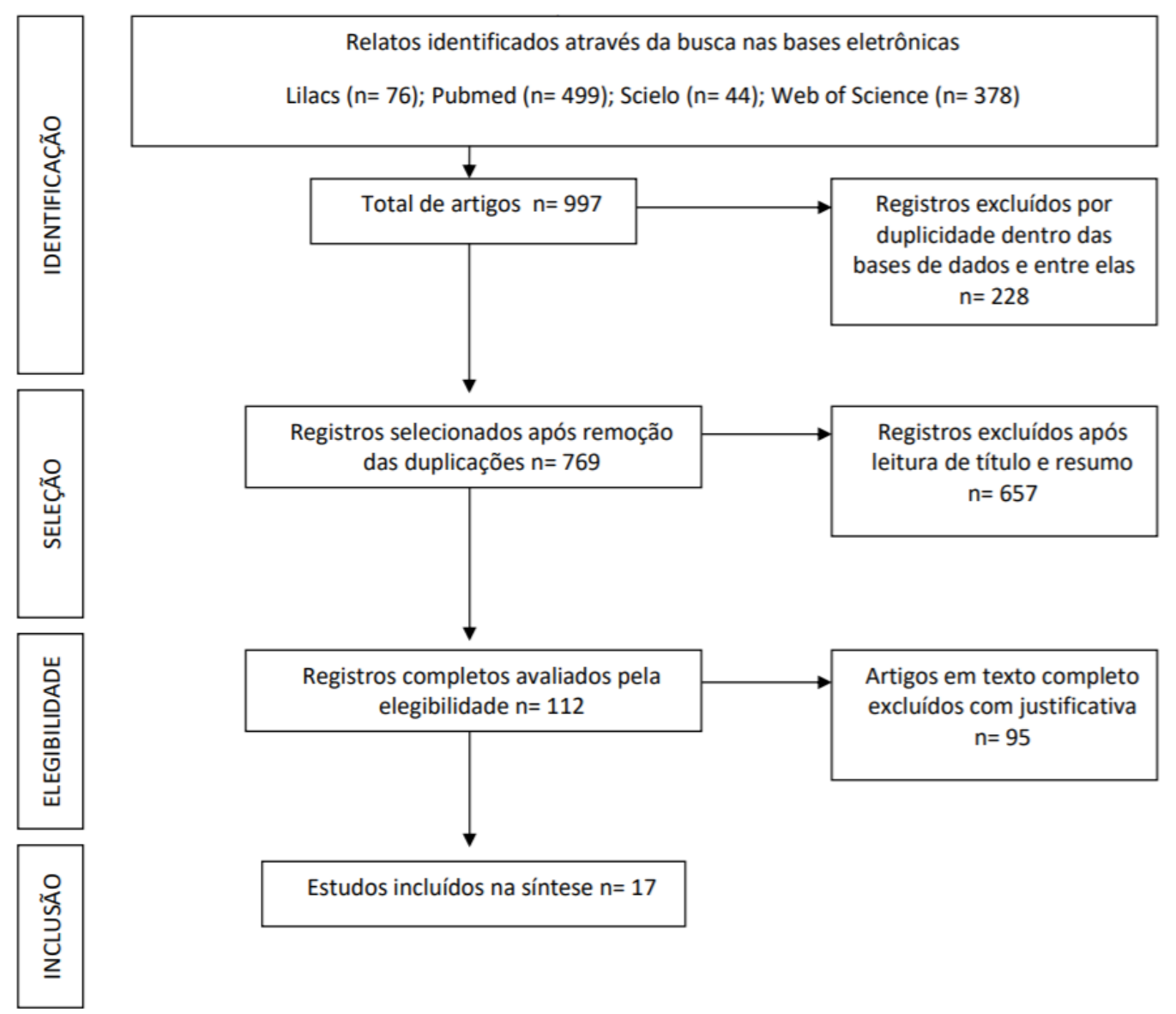

Fonte: Elaborado pelas autoras

Após a busca, os resultados foram exportados para o Software StArt (State of the Art Through Sistematic Review), desenvolvido pelo Laboratório de Pesquisa em Engenharia de Software (LaPES), da Universidade Federal de São Carlos (UFSCAR). O programa permite fazer o gerenciamento da revisão sistemática, desde o planejamento (protocolo), execução e sumarização, não havendo necessidade de se criar formulários adicionais.

\section{Resultados}

A busca nas bases eletrônicas resultou em 997 estudos; em seguida, procedeu-se a remoção de 228 artigos duplicados, resultando em 769 artigos. Após a leitura do título e do resumo, 657 foram excluídos por não atenderem aos critérios de inclusão, restando, assim, 112 trabalhos para serem analisados. A análise desses 112 trabalhos revelou que 17 cumpriam as exigências dos critérios de inclusão, sendo, assim, incluídos na presente revisão sistemática. O quadro 2 apresenta as características dos trabalhos selecionados. 


\section{Quadro 2 - Caracterização dos estudos sobre o envelhecimento ativo e os centros comunitários para idosos}

\begin{abstract}
Título do trabalho/local/Autor(es) e ano publicação

Grupo de convivência para idosos na atenção primária à saúde: contribuições para o envelhecimento ativo/ Brasil/ Previato et al., 2019.

Perfil sociodemográfico e clínico dos idosos de um Centro de Convivência/ Brasil/ Barbosa et al., 2018

Perfil dos Idosos do Centro-dia para Idosos "A Mão Branca" / Brasil/ Yamaguchi et al., 2018.

A esperança de vida dos idosos: avaliação pelo perfil e a Escala de Herth. Rev Fund Care/ Brasil/ Oliveira et al., 2018.

Qualidade de vida e nutrição em idosos participantes de centros de convivência/ Brasil/ Pereira; Filipe, 2017.

A sexualidade na velhice: representações sociais de idosos frequentadores de um grupo de convivência. Psicologia: Ciência e Profissão/ Brasil/ Vieira; Coutinho; Saraiva, 2016.
\end{abstract}

A participação masculina em grupos de convivência no contexto rural/ Brasil/Vargas; Portella, 2015.

Influência da orientação religiosa na qualidade de vida de idosos ativos/ Brasil/Barricelli; Silva; Araújo, 2012.

Vulnerability to AIDS among the elderly in an urban center in central Brazil/ Brasil/ Driemeier, 2012.

Significados da violência na velhice: narrativas e a autopercepção de idosos participantes em um centro de convivência no município de São Paulo (SP)/ Brasil/Araújo; Silva, 2011.

An Ethnography on the Healthy Life of the Aged Women Participating the Senior Centers/Coreia do Sul/Kim; Yang, 2018.

Motivation to volunteer among senior center participants/ Estados Unidos/Pardasani, 2018.

Perceived autonomy and self-care resources among senior center users/ Estados Unidos/ Matsui; Capezuti, 2008.

Practices and perceptions of food safety among seniors who prepare meals at home/

\section{Objetivos}

Analisar as contribuições do grupo de convivência de idosos para o envelhecimento ativo.

Delinear perfil sociodemográfico e clínico.

Traçar o perfil dos idosos.

Avaliar o nível de esperança de vida e traçar o perfil sociodemográfico, de saúde e riscos sociais dos idosos.

Traçar o perfil demográfico e nutricional e avaliar a qualidade de Vida e à satisfação com a saúde.

Investigar as representações sociais da sexualidade.

Identificar os motivos da adesão de homens idosos a participar do grupo de convivência.

Identificar modalidades de orientação religiosa, se intrínseca ou extrínseca, e possíveis relações com a qualidade de vida de idosos ativos, além de compreender as manifestações do fenômeno religioso para uma adequada atuação clínica nos desafios das práticas do atendimento ao idoso.

Avaliar a vulnerabilidade a Aids.

Identificar e descrever os significados da violência e maus-tratos contra a pessoa idosa.

Explorar padrões e significados de vida saudável entre mulheres.

Explorar a motivação para voluntariar.

Analisar a relação entre autonomia percebida e os recursos de autocuidado.

Determinar a necessidade de ações educativas relacionadas à segurança alimentar. 


\begin{tabular}{|c|c|}
\hline Estados Unidos/ Gettings; Kiernan, 2001 & \\
\hline $\begin{array}{l}\text { Bound by inequality: The social capital of older } \\
\text { Asians and Latinos in Phoenix, Arizona/ } \\
\text { Estados Unidos/Fukui; Menjivar, } 2015 .\end{array}$ & $\begin{array}{l}\text { Investigar a relação das interações cotidianas com } \\
\text { a geração de capital social, bem como os efeitos das } \\
\text { posições sociais. }\end{array}$ \\
\hline $\begin{array}{l}\text { Perceptions of Leisure by Older Adults Who } \\
\text { Attend Senior Centers/ Estados Unidos/ } \\
\text { Dattilo et al., 2015. }\end{array}$ & Verificar as percepções acerca do lazer. \\
\hline $\begin{array}{l}\text { Environments for Lifelong Learning in Senior } \\
\text { Centers/Estados Unidos/Eaton; Salari, } 2005\end{array}$ & $\begin{array}{l}\text { Examinar a influência do ambiente físico sobre as } \\
\text { atividades de aprendizagem ao longo da vida em } \\
\text { centros de idosos. }\end{array}$ \\
\hline
\end{tabular}

Fonte: Elaborado pelas autoras

Quanto ao local de realização dos estudos, verificou-se que 10 foram realizados no Brasil, 6 nos Estados Unidos e 1 na Coreia do Sul. Dentre os artigos produzidos no Brasil, 1 foi publicado em língua inglesa. As publicações se deram entre os anos de 2001 e 2019, sendo que o maior número de publicações (8) ocorreu em 2018, seguido por 3 publicações em 2015. Das 10 publicações de origem brasileira, 9 foram realizadas em instituições públicas e uma em instituição filantrópica. Os trabalhos oriundos dos Estados Unidos e Coreia do Sul nem sempre informam a natureza da instituição, mas, dentre os que informaram, houve predominância das instituições públicas.

Em relação à vinculação institucional dos autores dos artigos, verificou-se a expressiva participação de instituições de ensino superior como protagonistas das reflexões analisadas. Apenas um estudo foi conduzido por um grupo de profissionais sem vinculação com instituição de ensino.

A análise dos estudos revelou que a caracterização do centro, na maioria das vezes, inexistiu ou foi genérica, não sendo informados o número e idade dos idosos, tampouco as ações desenvolvidas nos centros comunitários e qual a relação do estudo com aquele grupo específico. De todo modo, dentre os que descreveram o centro, as atividades mais recorrentes foram lazer (viagem, dança sênior, coral, atividades socioculturais). O número de idosos foi informado em 3 trabalhos (PREVIATO et al., 2019; ARAÚJO; SILVA, 2011; PARDASANI, 2018), enquanto as ações puderam ser identificadas em 9 artigos (YAMAGUCHI et al., 2018; PREVIATO et al., 2019; ARAÚJO; SILVA, 2011; PARDASANI, 2018; PEREIRA; FILIPE, 2017; VIEIRA; COUTINHO; SARAIVA, 2016; VARGAS; PORTELLA, 2015; BARRICELI; SILVA; ARAÚJO, 2012; MATSUI; CAPEZUTI, 2008; FUKUI; MENJIVAR, 2015; EATON; SALARI, 2005). Nos artigos mencionados, as ações com maior presença nas instituições foram o lazer (7) e atividade física 
(6). As ações de saúde, educativas e de assistência social fazem parte do rol de serviços de 5, 4 e 3 centros, nessa ordem.

Em relação aos sujeitos envolvidos nos estudos foi verificado que as mulheres estiveram em maior número (70,58\%) em 12 estudos. Em 2 trabalhos, a amostra foi composta só por mulheres; em outro, foi voltada exclusivamente ao sexo masculino e em um contou com o mesmo número de mulheres e homens participando.

Os resultados sugeriram que o centro de convivência atuou como espaço de aprendizagem, contribuindo para a promoção da saúde e o envelhecimento ativo (PREVIATO et al., 2019); agiu no combate ao isolamento social (YAMAGUCHI et al., 2018; PEREIRA; FILIPE, 2017; OLIVEIRA et al., 2018); promoveu a incorporação da prática de atividade física ao estilo de vida pela maioria dos idosos (PEREIRA; FILIPE, 2017; BARRICELI; SILVA; ARAÚJO, 2012; BARBOSA et al., 2018); tinham maior participação feminina (YAMAGUCHI et al., 2018); influenciaram positivamente na saúde (KIM; YANG, 2018); fortaleceu os relacionamentos (KIM; YANG, 2018); e foi responsável pela oferta de serviços de cuidados para a maioria dos idosos (MATSUI; CAPEZUTI, 2008).

Em relação às condições de saúde, os estudos revelaram que os idosos apresentavam, pelo menos, uma doença crônica (YAMAGUCHI et al., 2018; BARBOSA et al., 2018); faziam uso de medicamentos (OLIVEIRA et al. 2018); apresentavam risco de desnutrição (PEREIRA; FILIPE, 2017); compreendiam que o comportamento sexual é o principal modo de propagação do HIV e reconheciam que o sexo sem proteção poderia deixá-los vulneráveis às doenças (DRIEMEIER et al., 2012); conjugaram práticas adequadas e inadequadas no preparo dos alimentos e precisavam de ações educativas que explicitassem a relação de microrganismos com a saúde (GETTINGS; KIERNAN, 2001).

No que se refere à qualidade de vida evidenciou-se que os idosos eram, em sua maioria, independentes em relação às atividades da vida diária (MATSUI; CAPEZUTI, 2008; OLIVEIRA et al. 2018); a esperança de vida é satisfatória para 78\% dos idosos estudados (OLIVEIRA et al. 2018); a sexualidade é ressignificada na velhice, passa a ser compreendida a partir do afeto, carinho, cumplicidade e ato sexual, sendo que este último perde a centralidade (VARGAS; PORTELLA, 2015); a maioria dos idosos declarou-se religiosa (BARRICELI; SILVA; ARAÚJO, 2012); estavam satisfeitos com sua qualidade de vida (BARRICELI; SILVA; ARAÚJO, 2012); a violência contra o idoso ocorre no âmbito familiar (ARAÚJO; SILVA, 2011); as justificativas e a importância do engajamento em trabalho voluntário, assim como a 
falta de motivação em fazê-lo não diferenciou dos idosos não frequentadores de centros de convivência (PARDASANI, 2018); o interesse pelo trabalho voluntário foi restrito (PARDASANI, 2018); os idosos demonstraram autonomia percebida positiva (MATSUI; CAPEZUTI, 2008); idosos com posições sociais privilegiadas favorecem a destinação de recursos para outros idosos e as desvantagens acumuladas ao longo da vida influenciam na formação de capital social (FUKUI; MENJIVAR, 2015); os idosos se mostraram resistentes à prática do lazer, o que exigiu a superação de desafios (DATTILO, 2015); a prática do lazer favoreceu a autodeterminação (DATTILO, 2015), e o modelo de educação ao longo da vida deve ser aquele que favoreça a interação como parte do processo educativo (EATON; SALARI, 2005).

\section{Discussão}

Esforços são percebidos nos estudos analisados no que se refere a ampliar a compreensão do fenômeno do envelhecimento para além dos aspectos biológicos, centrados apenas nas perdas. Assim, tem ganhado força a compreensão do envelhecimento como processo determinado pelo acesso, ou não, aos serviços sociais e de saúde, pelo ambiente físico, pelos recursos econômicos, pelo estilo de vida e pela genética. Todos esses fatores são atravessados pelas questões culturais e de gênero (OMS, 2005).

Os achados do estudo evidenciaram que há uma mobilização para ampliar a compreensão do envelhecimento. Foram acionados conceitos afetos aos pilares do envelhecimento ativo, que, por sua vez, estão imbricados com a qualidade de vida e promoção da saúde. Essa relação é explicitada nos títulos e objetivos dos estudos, e os aspectos inerentes à saúde, qualidade de vida e envelhecimento ativo aparecem em todos os trabalhos.

A despeito do envelhecimento populacional ser um fenômeno mundial típico de nações desenvolvidas, o Brasil experimenta o envelhecimento ainda de forma mais acelerada do que as nações desenvolvidas (MINAYO, 2012). Esse fato amplia os já conhecidos desafios impostos pelo envelhecimento e, nesse sentido, impulsiona os estudos voltados a sua compreensão.

Questões de gênero e culturais determinam o cuidado com a saúde e o estilo de vida, que, por sua vez, influenciam na expectativa de vida e longevidade de formas diferentes entre homens e mulheres. Assim, a presença expressiva de mulheres nos estudos é resultante do maior número de mulheres idosas e pela maior participação delas nos grupos de convivência (BARBOSA et al., 2018). A inserção masculina revela uma mudança de comportamento, em 
função de componentes culturais, que influenciam o seu envolvimento em novas atividades e por saberem que se trata de um espaço marcadamente feminino. Por outro lado, entende-se que o reconhecido domínio da população feminina nesses espaços pode influenciar no planejamento de ações que sejam capazes de atrair o público do sexo masculino (VARGAS; PORTELLA, 2015; PEREIRA; FILIPE, 2017).

Os centros de idosos são referência no Brasil e no mundo na missão de promover qualidade de vida à população idosa. Os centros têm atuado por meio de processos educativos, formativos, informativos e promotores, preventivos e curativos de saúde para neutralizar as perdas sociais, econômicas e físicas. Nesse sentido, os grupos de convivência desenvolvem programas de nutrição, lazer, educação e atividade física (PREVIATO et al., 2019; FUKUI; MENJIVAR, 2015; HAVIR, 1991).

Os resultados relacionados à saúde devem ser entendidos como reflexo de um estilo de vida ao longo da existência, conjugados com os processos naturais do envelhecimento (OLIVEIRA et al., 2018). O fato de vivenciarem doenças crônicas já foi referendado por diversos estudos (VERAS, 2012a; BARBOSA et al., 2018). Outras conclusões que ganham relevância no contexto deste estudo é que a população envelhecida demonstra comportamentos sexuais seguros para prevenir as infecções sexualmente transmissíveis. É preciso que o conhecimento assumido pelos idosos sobre as formas de exposição ao vírus, HIV, por exemplo, seja capaz de mudar o comportamento desses (DRIEMEIER et al., 2012). Nesse sentido, os achados apontam para a necessidade de ações de educação para saúde buscando desenvolver habilidades para o gerenciamento das doenças crônicas e adoção de práticas saudáveis.

As análises dos estudos evidenciaram a existência de gama variada de ações voltadas a promoção da qualidade de vida, entre as quais se destacaram a prática do lazer, a atividade física e a educação. Esses elementos perpassam as demais atividades dos centros, atuando na promoção da interação e da participação social, no desenvolvimento de habilidades, na diminuição da segregação social e na independência. Esses fatores podem impactar positivamente a saúde física e mental, a disponibilidade e a capacidade para se envolver em processos educativos, além de garantir a sensação de felicidade e satisfação com a vida (EATON; SALARI, 2005; DATTILO, 2015; CAVALCANTI et al., 2016).

Há o reconhecimento da importância desse equipamento na realidade norteamericana como ambientes que ofertam serviços direcionados à saúde e ao bem-estar da 
população envelhecida, entretanto, alertam sobre a escassez de estudos relacionando a melhoria da qualidade de vida e da saúde com a utilização dos centros (GIUNTA et al. 2012).

\section{Conclusão}

O reconhecimento da importância dos centros de convivência para idosos na promoção do envelhecimento ativo tornou-se fato a partir dos estudos analisados. São inúmeros os espaços de convivência orientados para ofertarem serviços visando minimizar e, ou, eliminar a segregação social, a perda de papéis sociais, a inatividade ou mesmo o sedentarismo. Além disso, esses lugares contribuem com processos educativos que estimulam a participação, promovem a saúde, auxiliam no gerenciamento de doenças crônicas e incentivam a adoção de hábitos saudáveis.

Este estudo procurou mapear e analisar as experiências dos centros comunitários de idosos na perspectiva do envelhecimento ativo, buscando contribuir para sinalizar a necessidade de pesquisas nesses espaços, com os idosos do centro, problematizando, principalmente, questões que emergiram de suas práticas, afastando, assim, a visão reducionista de que o centro atua como local de "recrutamento" de sujeitos para pesquisa.

Em função dos resultados dos estudos que envolveram idosos integrantes de centro comunitário para idoso, pode-se inferir que esse equipamento tem influenciado na saúde e na qualidade de vida de seus participantes, contribuindo, assim, para a efetivação da promoção do envelhecimento ativo.

Contudo, investigações que relacionam a organização e a dinâmica do centro com o atendimento aos idosos e os serviços e as estratégias metodológicas com a missão do centro têm sido pouco explorados. Muitas pesquisas realizadas com os sujeitos desses locais, geralmente, fazem parte de algum projeto de iniciação científica, de pesquisa de mestrado ou doutorado ou, ainda, estão relacionadas aos projetos de extensão universitária. Nesse sentido, é desejável que os centros, além de serem um locus para recepcionar pesquisas, também sejam indutores para as instituições de pesquisa pautarem temas de investigações. Dentre os diversos temas faz-se necessário compreender melhor esse equipamento como uma estratégia para ampliar a qualidade de vida e a ampliação da vida com qualidade.

Nesse sentido, faltam análises que demonstrem a sua efetividade e, quando necessário, indiquem pontos a serem corrigidos a partir da retroalimentação dos dados gerados pelas pesquisas, fornecendo fundamentos para elaboração de uma espécie de diretriz 
e correção de ações para esses espaços. Essa diretriz para o campo da pesquisa teria como ponto positivo o fato de ser resultante de uma empiria, e não construída fora do contexto de seu funcionamento, que envolve, em especial, os sujeitos atendidos, os idosos.

\section{Referências}

ARAÚJO, L. F.; SILVA, H. S. Significados da violência na velhice: narrativas e a autopercepção de idosos participantes em um centro de convivência no município de São Paulo (SP). Revista Kairós, v. 14, p. 05-29, 2011.

AZEVEDO, M. S. A. O Envelhecimento ativo e a qualidade de vida: uma revisão integrativa. Dissertação (Mestrado em Enfermagem Comunitária). Escola Superior de Enfermagem do Porto. Porto, 2015. Disponível em: https://comum.rcaap.pt/bitstream 10400.26/10776/1/marta\%2020\%20de\%20abril\%20-\%20tese\%20final\%20\%20pdf.pdf . Acesso em: jul. 2018.

BARBOSA, L. M.; NORONHA, K; SPYRIDES, M. H. C.; ARAUJO, C. A. D. Qualidade de vida relacionada à saúde dos cuidadores formais de idosos institucionalizados em Natal, Rio Grande do Norte. Rev. Bras. Est. Pop., Belo Horizonte, v. 34, n. 2, p. 391-414, maio/ago. 2017.

BARBOSA, R. L., SILVA, T. D. C. S., SANTOS, M. F; CARVALHO, F. R; MARQUES, R. V. D. A.; MATOS JUNIOR, E. M. Perfil sociodemográfico e clínico dos idosos de um Centro de Convivência. Revista Kairós-Gerontologia, v. 21, n. 2, p. 357-373, 2018.

BARRICELLI, I. L. F.O.B.L.; SILVA, L. M.; ARAUJO, C. V. Influência da orientação religiosa na qualidade de vida de idosos ativos. Rev. Brasileira de Geriatria e Gerontologia (UnATI. Impresso), v. 15, p. 505-515, 2012.

BRASIL. Ministério da Saúde. Secretaria-Executiva. Secretaria de Vigilância em Saúde. Glossário temático: promoção da saúde / Ministério da Saúde. Secretaria-executiva. Secretaria de Vigilância em Saúde. Brasília: Ministério da Saúde, 2012. 48 p. - (Série A. Normas e Manuais Técnicos).

CAVALCANTI, A. D.; MOREIRA, R. S.; BARBOSA, J. M. V.; SILVA, V. L. Envelhecimento ativo e estilo de vida: uma revisão sistemática da literatura. Estudos interdisciplinares do envelhecimento, Porto Alegre, v. 21, p. 71-89, 2016.

CENTRO INTERNACIONAL DE LONGEVIDADE BRASIL (CILB). Envelhecimento Ativo: um marco político em resposta à revolução da longevidade. Rio de janeiro: Centro Internacional de Longevidade Brasil, 2015.

DATTILO, J; LOREK, A. E.; MOGLE, J; SLIWINSKI, M.; FREED, S.; FRYSINGER M; SCHUCKERS, S. Perceptions of Leisure by Older Adults Who Attend Senior Centers, L. Scien.: An Interd. J., v. 37, n. 4, p. 373-390, 2015.

DRIEMEIER, M.; ANDRADE, S. M. O.; PONTES, E. R. J. C.; PANIAGO, A. M. M.; CUNHA, R. V. Vulnerability to AIDS among the elderly in an urban center in central Brazil. Clinics, v. 67, p. 
19-25, 2012.

EATON, J.; SALARI, S. Environments for Lifelong Learning in Senior Centers. Educational Gerontology, v. 31, n. 6, p. 461-480, 2005. DOI: 10.1080/03601270590928189.

FRANCISCO, C. M.; PINHEIRO, M. A. Espaços de convivência para idosos: benefícios e estratégias. Rev. Recien. São Paulo, v. 8, n. 24, p. 65-72, 2018.

FUKUI, H. M; MENJIVAR, C. Bound by inequality: The social capital of older Asians and Latinos in Phoenix. Ethnography, Arizonas, v. 16, n. 4, p. 416-437, 2015.

GETTINGS M. A; KIERNAN N.E. Practices and perceptions of food safety among seniors who prepare meals at home. Journal of nutrition education, v. 33, n. 3, p. 148-154, 2001.

GIUNTA, N.; MORANO, C.; PARIKH, N. S; FRIEDMAN, D.; FAHS. M. C; GALLO W. T. Racial and Ethnic Diversity in Senior Centers: Comparing Participant Characteristics in More and Less Multicultural Settings. Journal of Gerontological Social Work, v. 55, n. 6, p. 467-483, 2012.

HAVIR, L. Senior centers in rural communities: potentials for serving. Journal of aging studies, v. 5, n. 4, p. 359-374, 1991. Disponível em: https://www.sciencedirect.com Ljournal/journal-of-aging-studies. Acesso em: 25 mai. 2019.

KALACHE, A, VERAS, R. P.; RAMOS, L R. O envelhecimento da população mundial. Um desafio. Rev. Saúde Pública, v.21, n. 3, p. 200-210, 1987.

KIM, E. YANG, J. An Ethnography on the Healthy Life of the Aged Women Participating the Senior Centers. Journal of Korean Academy of Nursing, v. 48, n. 3, p. 349-361, 2018.

MARQUES, I. L. Atitudes face ao envelhecimento e percepção da qualidade de vida em indivíduos adultos mais velhos. 2017. Dissertação (Mestrado Integrado em Psicologia). Universidade de Lisboa, Faculdade de Psicologia, Lisboa, 2017.

MARTINS, R. C. C; CASETTO, J.S; GUERRA, R. L. F. Mudanças na qualidade de vida: a experiência de idosas em uma universidade aberta à terceira idade. Rev. Bras. Geriatra. Geront. [online], v. 22, n. 1, 2019.

MATSUI, M; CAPEZUTI E. Perceived autonomy and self-care resources among senior center users. Geriatric nursing, New York, v. 29, n. 2, p.141-147, 2008.

MINAYO, M. C. S. O envelhecimento da população brasileira e os desafios para o setor de saúde. Cadê. Saúde pública, Rio de janeiro, v. 28, n. 2, p. 208-209, 2012.

MOHER, D; LIBERATI, A; TETZLAFF, J; ALTMAN D.G, PRISMA Group. Preferred reporting items for systematic reviews and meta-analyses: the PRISMA statement. An Intern. Med, v. 151, n. 4, p. 264-269, 2009.

MOURA, M. M. D; VERAS, R. P. Acompanhamento do envelhecimento humano em centro de convivência. Physis [Internet]. v. 27, n. 1, p. 19-39, 2017. Disponível em: http://www.scielo.br/scielo.php?script=sci arttext\&pid=S0103-733120170001000 19\&Ing=en. DOI: http://dx.doi.org/10.1590/s0103-73312017000100002. Acesso em: 22 jun. 
2019.

OLIVEIRA, L. M.; SILVA, S. M.; LIMA, E. F. A.; GOMES, M. G. C.; OLYMPIO, P. C. A. P. A esperança de vida dos idosos: avaliação pelo perfil e a Escala de Herth. Rev Fund Care Online. v. 10, n. 1, p. 167-172, 2018. DOI: http://dx.doi.org/10.9789/2175-5361.2018.

ORGANIZAÇÃO MUNDIAL DA SAÚDE (OMS). Envelhecimento ativo: uma política de saúde/World Health Organization: Trad. Suzana Gontijo. Brasília: Organização Mundial PanAmericana de Saúde, 2005, 13p.

PARDASANI, M. Motivation to volunteer among senior center participants. Journal of Gerontological Social Work, v. 61, n. 3, p. 313-333, 2018. DOI: 10.1080/01634372.2018.1433259.

PEDRO, W. J. A. Processos de envelhecimento em Portugal. Usos do tempo, redes sociais e condições de vida. Rev. Kairós Gerontologia, São Paulo, v. 16, n.3, pp.331-334, jun. 2013. Disponível em: http://www.ffms.pt/upload/docs/4e326275-8ec4-4e10-8e5dOdab9ad2657a.pdf. Acesso em: 20 mar. 2018.

PEREIRA, M. M; FILIPE, E. M. V. Qualidade de vida e nutrição em idosos participantes de centros de convivência. Revista Baiana de Saúde Pública, [S.I.], v. 40, n. 1, set. 2017. ISSN 2318-2660. Disponível em: http://rbsp.sesab.ba.gov.br/index.php/rbsp/article /view/ 1615 . 2019. DOI: https://doi.org/10.22278/2318-2660.2016. V 4 0.n1.a1615. Acesso em: 23 jun.

PEREIRA, M. M; FILIPE, E. M. V. Qualidade de vida e nutrição em idosos participantes de centros de convivência. Revista Baiana de Saúde Pública, [S.I.], v. 40, n. 1, set. 2017. ISSN 2318-2660. Disponível em: <http://rbsp.sesab.ba.gov.br/index.php/rbsp/article/view L1615>. DOI: https://doi.org/10.22278/2318-2660.2016. v40.n1.a1615. Acesso em: 23 jun. 2019.

PREVIATO G. F; NOGUEIRA, I. S, MINCOFF, R. C. L; JAQUES, A. E; CARREIRA, L; BALDISSERA, V. D. A. Grupo de convivência para idosos na atenção primária à saúde: contribuições para o envelhecimento ativo. Rev. Fun. Care Online, v. 11, n. 1, p. 173-180, 2019. DOI: http://dx.doi.org/10.9789/2175-5361.2019.v11i1.173-180.

REIS, C. S.; NORONHA, K. WAJNMAN, S. Envelhecimento populacional e gastos com internação do SUS: uma análise realizada para o Brasil entre 2000 e 2010. Rev. bras. estud. Popul., São Paulo, v. 33, n. 3, p. 591-612, dez. 2016. Disponível em: http://www.scielo.br/scielo.php?script=sci arttext\&pid=S0102-30982016000300591 \&lng=pt\&nrm=iso. Acesso em: 22 abr. 2018.

REIS, C; BARBOSA, L. M. L. H; PIMENTEL, V. P. O desafio do envelhecimento populacional na perspectiva sistêmica da saúde. BNDES Setorial, Rio de Janeiro, n. 44, p. 87-124, 2016.

VARGAS, A. C.; PORTELLA, M. R. A participação masculina em grupos de convivência no contexto rural. Estudos Interdisciplinares sobre o Envelhecimento, v. 20, p. 485-494, 2015.

VELOSO, A. S. T. Envelhecimento, saúde e satisfação - efeitos do envelhecimento ativo na qualidade de vida. 2015. Dissertação (Mestrado em Gestão e Economia da Saúde) Universidade de Coimbra - Faculdade de Economia, Coimbra, 2015. 
VERAS, R. P. Prevenção de doenças em idosos: os equívocos dos atuais modelos. Cad. Saúde Pública [online], v. 28, n. 10, p. 1834-1840, 2012.

VERAS, R. P. Gerenciamento de doença crônica: equívoco para o grupo etário dos idosos. Rev. Saúde Pública, v. 46, n. 6, p. 929-934, 2012a.

VIEIRA, K. F. L.; COUTINHO, M. P. L.; SARAIVA, E. R. A. A sexualidade na velhice: representações sociais de idosos frequentadores de um grupo de convivência. Psicologia: Ciência e Profissão (Online), v. 16, p. 196-209, 2016.

YAMAGUCHI, M. B; SILVA, J; LINS, C. L., CONCEIÇÃO, M. F; OLIVEIRA, S. S; CAMASMIE, A; CHADE, L. D. Perfil dos Idosos do Centro-dia para Idosos "A Mão Branca". Revista KairósGerontologia, v. 21, n. 2, p. 393-405, 2018.

YAMAMOTO, C. M. K. Promoção da saúde do idoso: revisão de literatura brasileira. 2010. Dissertação (Mestrado em Saúde Pública) - Universidade de São Paulo - Faculdade de Saúde Pública, São Paulo, SP, 2010. 